\title{
INFLUENCE OF INTERFACE GAP ON \\ THE STRESS BEHAVIOUR OF SMART SINGLE LAP JOINTS UNDER TIME HARMONIC LOAD
}

\author{
JORDANKA IVANOVA*, VARBINKA VALEVA \\ Institute of Mechanics, Bulgarian Academy of Sciences, Acad. G. Bonchev \\ Str., bl. 4, 1113 Sofia, Bulgaria
}

[Received 15 May 2017. Accepted 26 June 2017]

\begin{abstract}
Adhesive joints are frequently used in different composite structures due to their improved mechanical performance and better understanding of the failure mechanics. The application of such structures can be seen in aerospace and high technology components.

The authors developed and applied modified shear lag analysis to investigate the hygrothermalpiezoelectric response of a smart single lap joint at environmental conditions (with/without an interface gap along the overlap zone) and under dynamic time harmonic mechanical and electric loads. The main key is the study of the appearance of possible delamination along the interface. As illustrative examples, the analytical closed form solution of the structure shear and the axial stresses response, as well as the interface debond length, including influence of mechanical, piezoelectric, thermal characteristics and frequencies is performed and discussed. All results are presented in figures. The comparison of the shear stress and electric fields for both cases of overlap zone (continuous or with a gap) is also shown in figures and discussed.
\end{abstract}

KEY WORDS: Smart single lap joint, dynamic time harmonic loading, shear lag method, interface debond length, hygropiezothermal behaviour.

\section{INTRODUCTION}

Joints are the one of the most used, but they are one of the complex parts of a structural assembly, because they create discontinuities in the geometry of the structures and the material properties. Many catastrophic structural failures, emanated from joints are observed in engineering practice.

Joints, used in aeronautic and space constructions, can be divided into joints fastened by mechanical means such as bolts rivets or screws, adhesively bonded joints or both adhesively bonded and fastened.

The classical paper [1] is the most cited work in the analysis of adhesively bonded joints. It analyzed a single-lap joint for two limiting cases. During the analysis it was

*Corresponding author e-mail: ivanova@imbm.bas.bg 
assumed that (a) the axial stress in the adhesive layer can be neglected, and (b) normal and transverse shear stress in the adhesive layer do not vary across the thickness of the adhesive. These basic assumptions have been employed by numerous authors to extend the work in the area of analysis and design of bonded joints. The work [2] is a continuation of the work [1], where the variation of the stress across the thickness of the adhesive has been considered. In [3], the extensive analytical work in the area of adhesively bonded joints, involving composite adherents, was carried out. Introducing Vokersen's shear lag equation, that calculates shear stress in the adhesive, the strength for lap joints, especially with composite adherends by classical linear elastic solution was predicted in [4,5].

The analytical analysis of adhesively bonded joints was simplified in most cases. The reason for simplification is the large number of the equations that had to be carried through the analysis, and the needed long time to complete the analysis.

For the past three decades, researchers and engineers have been involved in the development of various numerical techniques to analyze different kinds of bonded joints in composite structures. One of the computer codes was proposed in [6]. The eight-node elements, in order to manage high stress gradients at the interface analyzing the single lap joint, thick adherend specimen and crack-lap joint were used. In [7,8], a non-linear finite element method was implemented to predict the strength of a bonded single-lap joint and considered the strength of steel lap joints, loaded in tension and performed a detailed stress analysis of the shear and the transverse stresses in the joint.

The influence of the bond line thickness and the overlap length on the strength of bonded joints was investigated in [9]. In [10], a single-lap joint with laminated polymeric composite adherends and with a spew fillet, subjected to tensile loading was analyzed. The finite element analysis was also used for this problem to address the mechanics and the deformation of such material and bonding configuration.

Extensive research on the stress singularity near the vertex of a bi-material wedge has been conducted in [11-15]. Authors analyzed the plane problem of a composite body, consisting of many dissimilar isotropic, homogeneous, and elastic wedges, perfectly bonded along their common interfaces.

One of the issues in adhesive bonded joints is the prediction of failure mechanisms. It has been assumed in most literature, that failure initiation criterion is stressbased, strain-based or displacement based $[16,17]$. It has been observed, that failure of an adhesively bonded joint depends upon the crack initiation position.

The focus of the present study is the adhesive failure between adhesive and adherent, where crack initiates and propagates along the interface. Some effects, as flaws and voids in adhesive bonded joints statically loaded have been studied in [18,19].

The authors consider a smart single lap joint under dynamic time harmonic me- 
chanical and electric loads at environment conditions. The analytical solution is obtained, using shear lag analysis. The solutions for the cases of continuous interface of a joint and a joint with a gap, along the interface are obtained. The overlap region for the case of joint with an interfacial gap is divided into three zones: one is zone with a gap and two are zones of the continuous joint. Assuming, that the first layer has piezoelectric properties (PZT-4) and the second one has elastic properties (IM7), the respective calculations of debond lengths for the cases with and without interfacial gap are performed. The influence of temperature and moisture is taken into account. The obtained results for interface shear stress, debond length and electric are illustrated in figures and discussed.

\section{StATEMENT OF THE PROBLEM}

In this paper, the dynamic time harmonic behaviour of a smart single lap joint at environment conditions, under dynamic time harmonic and constant electrical loads will be considered (Fig. 1). The shear lag method [16] will be used for this purpose.

Within the engineering society, this method became a common computational tool for analytical stress analysis in composite materials, adhesive joints, etc. The main idea of the shear-lag analysis is a simplification of in-plane shear stress decoupling the $2 \mathrm{D}$ problem into two $1 \mathrm{D}$ ones, due to the hypothesis, that the load is transferred from the adhesive interface to the adjacent ones by the matrix shear force. Hence, the matrix shear force is independent on the transverse displacements.

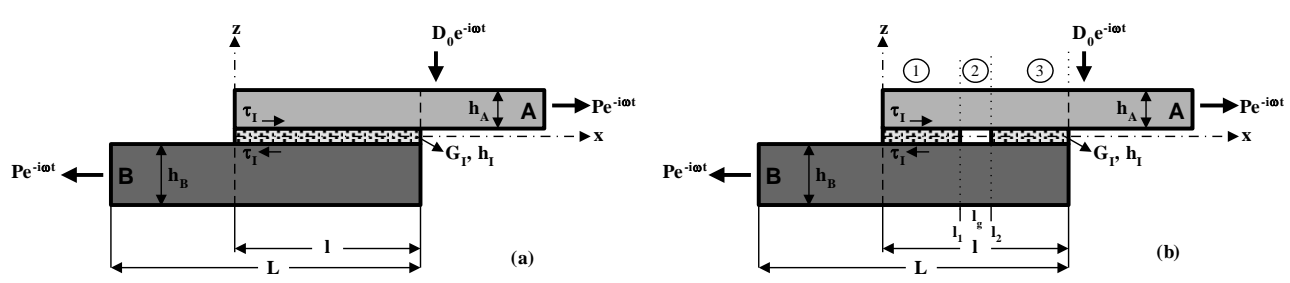

Fig. 1. (a) Continuous joint and (b) joint with a gap under tensile time harmonic load.

According to Fig. $1 h_{\alpha}(\alpha=A, B, I)$ are the thicknesses of the adherents $A, B$ and interface $I$ and $l$ is the length of the overlap zone. For the case of a single lap joint with a gap (Fig. $1 \mathrm{~b}$ ), the interface is divided into three zones with the lengths $l_{1}=l_{I}, l_{g}=\left(l_{2}-l_{1}\right)$ and $l_{3}=\left(l-l_{2}\right)$. The external load consists in applied dynamic time harmonic extensional load $P e^{-i \omega t}$ and electric field with a poling axis $O z$, given by the electric displacement $D_{0} e^{-i \omega t}$. The importance of such an approach is connected with the large shear stresses, arising in the single lap joints. According to materials used in such structure, it is assumed, that in general, the first layer $A$ is transversal isotropic elastic with piezoelectric properties and sensitive to thermal 
effects. The second layer $B$, in general, is transversally isotropic, but sensitive to thermal and moisture effects. Both adherends are connected with an isotropic elastic interface layer with a shear modulus $G_{I}$. The interface works only on shear,while both adherends work on extension. Any bending is neglected.

Due to the character of the applied combined load and using the Fourier method, all parameters of the problem stated are presented as follows:

$$
\begin{array}{lll}
u_{\alpha}(x, t)=u_{\alpha}(x) e^{-i \omega t} & \sigma_{\alpha}(x, t)=\sigma_{\alpha}(x) e^{-i \omega t} & \\
\tau_{I}(x, t)=\tau_{I}(x) e^{-i \omega t} & T_{\alpha}(x, t)=T(x) e^{-i \omega t} & (\alpha=A, B) \\
D_{z A}(x, t)=D_{z A}(x) e^{-i \omega t} & E_{z A}(x, t)=E_{z A}(x) e^{-i \omega t} & H_{B}(x, t)=H(x) e^{-i \omega t},
\end{array}
$$

where $u_{\alpha}, \sigma_{\alpha}, \tau_{I}(\alpha=A, B)$ denote the displacements, stresses and interfacial shear stress of the considered structure at time harmonic mechanical and electric field. $E_{z A}$ and $D_{z A}$ are the $z$-components of the electric field and displacement of layer $A$; $T$ and $H$ are the temperature and moisture concentration in the structure, $\omega$ is the frequency. Further in the paper the z-component of the electric field $E_{z A}$ is called electric gradient.

\section{Problem SOlution}

The constitutive equations for the posed problem are

$$
\begin{gathered}
\sigma_{A}=\left(c_{11}^{A *}+\frac{e_{13}^{A *} e_{31}^{A *}}{\varepsilon_{33}^{A *}}\right) \varepsilon_{A}-\frac{e_{13}^{A *}}{\varepsilon_{33}^{A *}} D_{0}-\left(\alpha_{11}^{A *}-\frac{e_{13}^{A *}}{\varepsilon_{33}^{A *}} p_{3}^{A *}\right) T \\
\sigma_{B}=c_{11}^{B *} \varepsilon_{B}-\alpha_{11}^{B *} T-\beta_{11}^{B *} H \quad \tau_{I}=\frac{G_{I}}{h_{I}}\left(u_{A}^{(2)}-u_{B}^{(2)}\right) \\
E_{z A}=\frac{D_{0}}{\varepsilon_{33}^{A *}}-\frac{e_{31}^{A *}}{\varepsilon_{33}^{A *}} \varepsilon_{A}-\frac{p_{3}^{A *}}{\varepsilon_{33}^{A *}} T,
\end{gathered}
$$

where $\alpha_{11}^{A, B}, \beta_{11}^{B}$ are the thermal and moisture expansion coefficients. For more details about the determination of the coefficients with ' $*$ ' see [20].

For simplicity, we introduce the following notations:

$$
\begin{aligned}
& E_{A}=\left(c_{11}^{A *}+\frac{e_{13}^{A *} e_{31}^{A *}}{\varepsilon_{33}^{A *}}\right), \quad D_{A}=\frac{e_{13}^{A *}}{\varepsilon_{33}^{A *}} D_{0}, \quad \alpha_{A}=\left(\alpha_{11}^{A *}-\frac{e_{13}^{A *}}{\varepsilon_{33}^{A *}} p_{3}^{A *}\right), \\
& E_{B}=c_{11}^{B *}, \quad \alpha_{B}=\alpha_{11}^{B *}, \quad \beta_{B}=\beta_{11}^{B *} .
\end{aligned}
$$

For the case of continuous joint 2D dynamic equations, resulting from the hypothesis of the shear lag method, we have the following form: 


$$
\begin{array}{ll}
\frac{d \sigma_{A}}{d x}-\frac{\tau_{I}}{h_{A}}=\rho_{A} \frac{\partial^{2} u_{A}}{\partial t^{2}} & \frac{d \sigma_{B}}{d x}+\frac{\tau_{I}}{h_{B}}=\rho_{B} \frac{\partial^{2} u_{B}}{\partial t^{2}} \\
\tau_{I}=\frac{G_{I}}{h_{I}}\left(u_{A}-u_{B}\right) & \frac{d D_{z A}}{d z}=0 \quad\left(D_{z A}=D_{0}\right),
\end{array}
$$

where $\rho_{A}, \rho_{B}$ are the densities of the respective material of layers.

Using constitutive equations (1), the first two equations of (3) regard as

$$
\begin{aligned}
\frac{d^{2} u_{A}}{d x^{2}}-\frac{G_{I}}{h_{I} E_{A} h_{A}}\left(u_{A}-u_{B}\right) & =-\frac{\rho_{A}}{E_{A}} \omega^{2} u_{A}, \\
\frac{d^{2} u_{B}}{d x^{2}}+\frac{G_{I}}{h_{I} E_{B} h_{B}}\left(u_{A}-u_{B}\right) & =-\frac{\rho_{B}}{E_{B}} \omega^{2} u_{B} .
\end{aligned}
$$

The following parameters are additionally introduced:

(5) $\xi=\frac{\rho_{A}}{E_{A}} \omega^{2}, \quad \eta=\frac{\rho_{B}}{E_{B}} \omega^{2}, \quad \varsigma=\frac{E_{A}}{E_{B}}, \quad \kappa=\frac{1}{h_{I} h_{A}} \frac{G_{I}}{E_{A}}, \quad \lambda=\frac{1}{h_{B} h_{I}} \frac{G_{I}}{E_{B}}$.

Then, (4) takes the form

$$
u_{A}^{\prime \prime}-\kappa\left(u_{A}-u_{B}\right)=-\xi u_{A}, \quad u_{B}^{\prime \prime}+\lambda\left(u_{A}-u_{B}\right)=-\eta u_{B} .
$$

After some transformation, the system of ordinary differential equations (6) is reduced to the following $4^{\text {th }}$ order differential equation, about the axial displacement of the layer $A$ :

$$
u_{A}^{I V}-A u_{A}^{\prime \prime}-B u_{A}=0
$$

where

$$
A=k-\xi+\lambda-\eta, \quad B=\kappa \eta-\xi \eta+\lambda \xi \quad \text { and } \quad u_{B}=\frac{1}{k}\left[(k-\xi) u_{A}-u_{A}^{\prime \prime}\right] .
$$

The respective characteristic equation of (7) is

$$
r^{4}-A r^{2}-B=0, \quad r^{2}=z, \quad z^{2}-A z-B=0
$$

with roots $z_{1,2}=\left(A \pm \sqrt{A^{2}+4 B}\right) / 2$. The following solutions take place:

\section{SOLUTION 1:}

$$
z_{1}>0, z_{2}>0 \Rightarrow r_{1,2}=p= \pm \sqrt{z_{1}}, \quad r_{3,4}=q= \pm \sqrt{z_{2}}
$$




$$
\begin{aligned}
u_{A}= & A_{1} \cosh (p x)+A_{2} \sinh (p x)+A_{3} \cosh (q x)+A_{4} \sinh (q x), \\
u_{B}= & \frac{\left(k-\xi-p^{2}\right)}{k}\left[A_{1} \cosh (p x)+A_{2} \sinh (p x)\right] \\
& +\frac{\left(k-\xi-q^{2}\right)}{k}\left[A_{3} \cosh (q x)+A_{4} \sinh (q x)\right] .
\end{aligned}
$$

\section{SOLUTION 2:}

$$
\begin{aligned}
z_{1}>0, z_{2}<0 \Rightarrow r_{1,2}=p= \pm \sqrt{z_{1}}, \quad r_{3,4}=q= \pm i \sqrt{\left|z_{2}\right|}, \\
z_{1}<0, z_{2}>0 \Rightarrow r_{1,2}=p= \pm \sqrt{z_{2}}, \quad r_{3,4}=q= \pm i \sqrt{\left|z_{1}\right|}, \\
u_{A}=A_{1} \cosh (p x)+A_{2} \sinh (p x)+A_{3} \cos (q x)+A_{4} \sin (q x) \\
u_{B}=\frac{\left(k-\xi-p^{2}\right)}{k}\left[A_{1} \cosh (p x)+A_{2} \sinh (p x)\right] \\
+\frac{\left(k-\xi+q^{2}\right)}{k}\left[A_{3} \cos (q x)+A_{4} \sin (q x)\right] .
\end{aligned}
$$

\section{SOLUTION 3:}

$$
\begin{aligned}
z_{1}<0, & z_{2}<0 \Rightarrow r_{1,2}=p= \pm i \sqrt{\left|z_{1}\right|}, \quad r_{3,4}=q= \pm i \sqrt{\left|z_{2}\right|}, \\
u_{A}= & A_{1} \cos (p x)+A_{2} \sin (p x)+A_{3} \cos (q x)+A_{4} \sin (q x), \\
u_{B}= & \frac{\left(k-\xi+p^{2}\right)}{k}\left[A_{1} \cos (p x)+A_{2} \sin (p x)\right] \\
& +\frac{\left(k-\xi+q^{2}\right)}{k}\left[A_{3} \cos (q x)+A_{4} \sin (q x)\right]
\end{aligned}
$$

The dynamic equations for the gap zone (2), where the shear stress is zero $\left(\tau_{I}=0\right)$, i.e. $u_{A}=u_{B}$, possess the form

$$
\frac{d^{2} u_{A}}{d x^{2}}=-\xi u_{A}, \quad \frac{d^{2} u_{B}}{d x^{2}}=-\eta u_{B} .
$$

The characteristic equation $r^{2}=-(\xi+\eta) / 2$ possesses roots $r_{1,2}= \pm i \sqrt{(\xi+\eta) / 2}$ and the following solutions take place:

$$
u_{A}=u_{B}=A_{1} \cos (r x)+A_{2} \sin (r x) .
$$

To find the unknown constants in the solution, the following boundary and contact conditions are taken into account as follows:

- for a continuous joint 


$$
\sigma_{A}(0)=0, \quad \sigma_{A}(l)=\frac{P}{w h_{A}}, \quad \sigma_{B}(0)=\frac{P}{w h_{B}}, \quad \sigma_{B}(l)=0
$$

- for a joint with a gap (here and below the upper index in brackets denotes the number of zone for the case of a joint with a gap

$$
\begin{array}{cc}
\sigma_{A}^{(1)}(0)=0, \quad \sigma_{B}^{(1)}(0)=\frac{P}{w h_{B}}, & \sigma_{A}^{(3)}(l)=\frac{P}{w h_{A}}, \quad \sigma_{B}^{(3)}(l)=0 \\
u_{A}^{(1)}\left(l_{1}\right)=u_{B}^{(1)}\left(l_{1}\right), & u_{A}^{(3)}\left(l_{2}\right)=u_{B}^{(3)}\left(l_{2}\right) \\
\sigma_{A}^{(1)}\left(l_{1}\right)=\sigma_{A}^{(2)}\left(l_{1}\right), & \sigma_{B}^{(1)}\left(l_{1}\right)=\sigma_{B}^{(2)}\left(l_{1}\right) \\
\sigma_{A}^{(3)}\left(l_{2}\right)=\sigma_{A}^{(2)}\left(l_{2}\right), & \sigma_{B}^{(3)}\left(l_{2}\right)=\sigma_{B}^{(2)}\left(l_{2}\right)
\end{array}
$$

We combine the equilibrium equations and stress-strain equations [18], in order to derive the continuity equation over the gap region. Consider the strain

$$
\begin{aligned}
& \varepsilon_{A}=\frac{\partial u_{A}}{\partial x}+\frac{1}{E_{A}}\left(D_{A}+\alpha_{A} T\right), \\
& \varepsilon_{B}=\frac{\partial u_{B}}{\partial x}+\frac{1}{E_{B}}\left(\alpha_{B} T+\beta_{B} H\right), \\
& \varepsilon_{I}=\frac{\varepsilon_{A}+\varepsilon_{B}}{2}
\end{aligned}
$$

i.e. the axial strain in the interface $\varepsilon_{I}$ is the average of the axial strains in layers $A$ and $B$.

Using equations (15) and integrating, we have

$$
\begin{aligned}
\int_{l_{1}}^{l_{2}} \varepsilon_{A} d x & =\int_{l_{1}}^{l_{2}} d u_{A}+\frac{1}{E_{A}}\left(D_{A}+\alpha_{A} T\right) \int_{l_{1}}^{l_{2}} d x \\
& =u_{A}\left(l_{2}\right)-u_{A}\left(l_{1}\right)+\frac{\left(D_{A}+\alpha_{A} T\right)}{E_{A}}\left(l_{2}-l_{1}\right) \\
\int_{l_{1}}^{l_{2}} \varepsilon_{B} d x & =\int_{l_{1}}^{l_{2}} d u_{B}+\frac{1}{E_{B}}\left(\alpha_{B} T+\beta_{B} H\right) \int_{l_{1}}^{l_{2}} d x \\
& =u_{B}\left(l_{2}\right)-u_{B}\left(l_{1}\right)+\frac{\left(\alpha_{B} T+\beta_{B} H\right)}{E_{B}}\left(l_{2}-l_{1}\right) .
\end{aligned}
$$

Above equations involve the change in the displacements at the interface, as we go from $l_{1}^{-}$to $l_{2}^{+}$over the gap region. 
Using the equations (4), the following relations can be obtained:

$$
\frac{1}{E_{A}} \int_{l_{1}}^{l_{2}}\left(\sigma_{A}+D_{A}+\alpha_{A} T\right) d x=u_{A}\left(l_{2}\right)-u_{A}\left(l_{1}\right)+\frac{\left(D_{A}+\alpha_{A} T\right)}{E_{A}}\left(l_{2}-l_{1}\right),
$$

$$
\frac{1}{E_{B}} \int_{l_{1}}^{l_{2}}\left(\sigma_{B}+\alpha_{B} T+\beta_{B} H\right) d x=u_{B}\left(l_{2}\right)-u_{B}\left(l_{1}\right)+\frac{\left(\alpha_{B} T+\beta_{B} H\right)}{E_{B}}\left(l_{2}-l_{1}\right),
$$

$$
\begin{aligned}
& \left.\frac{d \sigma_{A}}{d x}\right|_{l_{1}^{-}}=\left.\frac{G_{I}}{h_{I} h_{A}}\left(u_{A}-u_{B}\right)\right|_{l_{1}}-\left.\rho_{A} \omega^{2} u_{A}\right|_{l_{1}}, \\
& \left.\frac{d \sigma_{A}}{d x}\right|_{l_{2}^{+}}=\left.\frac{G_{I}}{h_{I} h_{A}}\left(u_{A}-u_{B}\right)\right|_{l_{2}}-\left.\rho_{A} \omega^{2} u_{A}\right|_{l_{2}}, \\
& \left.\frac{d \sigma_{B}}{d x}\right|_{l_{1}^{-}}=-\frac{G_{I}}{h_{I} h_{B}}\left(u_{A}-u_{B}\right)\left|l_{1}-\rho_{B} \omega^{2} u_{B}\right|_{l_{1}}, \\
& \left.\frac{d \sigma_{B}}{d x}\right|_{l_{2}^{+}}=-\left.\frac{G_{I}}{h_{I} h_{B}}\left(u_{A}-u_{B}\right)\right|_{l_{2}}-\left.\rho_{B} \omega^{2} u_{B}\right|_{l_{2}} .
\end{aligned}
$$

Combining equations (17) and (18), we obtain

$$
\begin{aligned}
& \left.\frac{d \sigma_{A}^{(3)}}{d x}\right|_{l_{2}}-\left.\frac{d \sigma_{A}^{(1)}}{d x}\right|_{l_{1}}=(\kappa-\xi) \int_{l_{1}}^{l_{2}} \sigma_{A}^{(2)} d x_{A}-k \varsigma \int_{l_{1}}^{l_{2}} \sigma_{B}^{(2)} d x, \\
& \left.\frac{d \sigma_{B}^{(3)}}{d x}\right|_{l_{2}}-\left.\frac{d \sigma_{B}^{(1)}}{d x}\right|_{l_{1}}=-\frac{\lambda}{\varsigma} \int_{l_{1}}^{l_{2}} \sigma_{A}^{(2)} d x+(\lambda-\eta) \int_{l_{1}}^{l_{2}} \sigma_{B}^{(2)} d x .
\end{aligned}
$$

After applying conditions (13), (14) and (19), we obtain the expressions for constants, which are presented in the Appendix.

The length of the interfacial debonding $l_{e}$ can be found from the condition $\tau_{I}\left(x_{d}\right)=\tau^{c r}$.

\section{NUMERICAL EXAMPLE}

For the numerical example, the following geometry characteristics are used: $h_{A}=$ $2 \mathrm{~mm}, h_{B}=4 \mathrm{~mm}, h_{I}=0.2 \mathrm{~mm}, l=30 \mathrm{~mm}, w=10 \mathrm{~mm}, l_{g}=4 \mathrm{~mm}, l_{1}=$ $5.13 .21 \mathrm{~mm}, G_{I}=800 \mathrm{MPa}, \tau^{c r}=18 \mathrm{MPa}, D_{0}=0.01 \mathrm{C} / \mathrm{m}^{2}, T=310 \mathrm{~K}$, $H=5$ wt. $\%, P=1 \div 50 \mathrm{kN}, \omega=0.1 \div 1 \mathrm{MHz}$. The mechanical characteristics of used materials are given in [20]. 
The following cases are considered:

Case 1: $\quad \alpha_{A}=0, \quad \alpha_{B}=0, \quad \beta_{B}=0$

Case 2: $\quad \alpha_{A} \neq 0, \quad \alpha_{B} \neq 0, \quad \beta_{B} \neq 0$.

Figures 2-4 show the behaviour of the interface shear stress, electric gradient and values of debond length, along the overlap region for a continuous joint (a) and a joint with a gap (b), located on the left side of the overlap at fixed parameters $D_{0}$, $T, H$ for cases 1, 2 and three values of frequency. Figures also show the influence of mechanical characteristics of the layer $B$ and the presence of temperature $[12,13]$ and moisture.

In Fig. 2, the behaviour of the interface stress along the overlap length zone is shown. The comparison of the interface shear stress for the case 1 and 2 shows higher values of the interface shear stress for case 1 and at higher amplitude of the external load. The presence of a gap at the left of the overlap zone leads to the decreasing
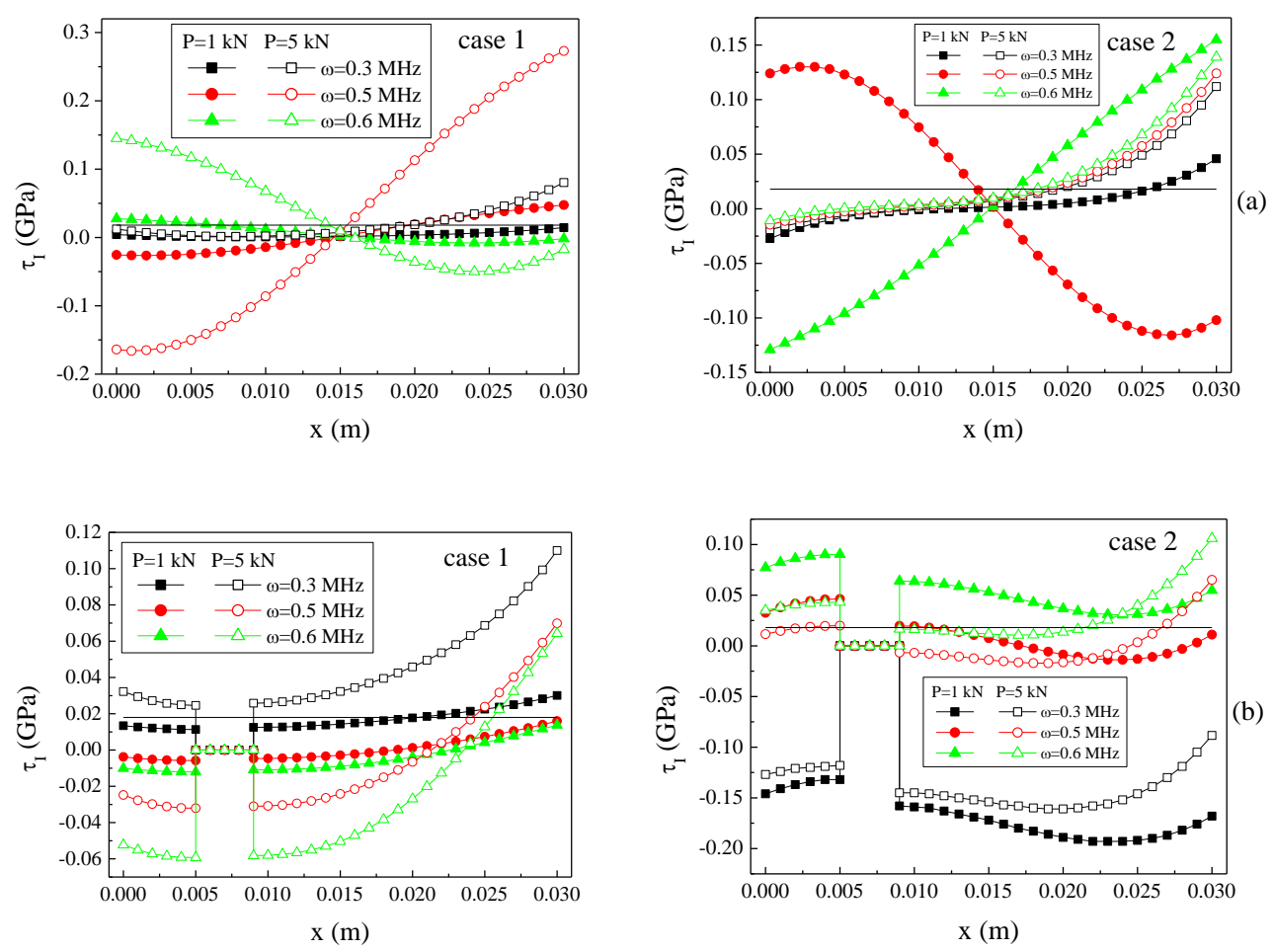

Fig. 2. Shear stress along the interface of the overlap length of the joint: (a) for a continuous joint; (b) for a joint with a gap. 

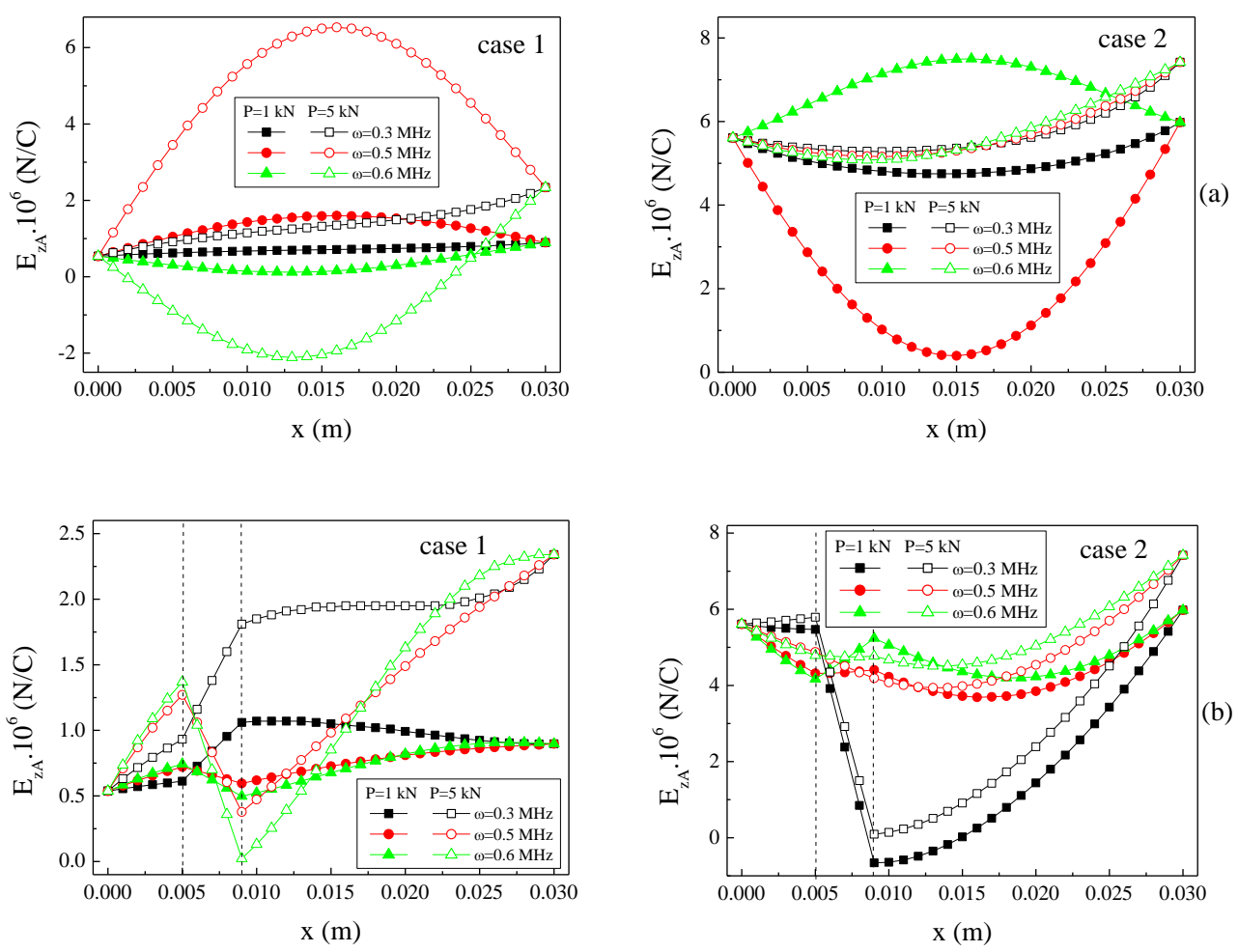

Fig. 3. Electric gradient along the overlap length of the joint: for a continuous joint; (b) for a joint with a gap.

in interfacial shear stresses. The straight line $\tau=\tau^{c r}$ intersects the function of the interface shear stress at left or right positions $x_{d}$, which indicates the appearance of the debond length from the left or right sides of the overlap zone.

In Fig. 3 the behaviour of the electric gradient, coming from the layer $A$ along overlap zone, is shown. The presence of the temperature and moisture, as well as the values of frequency and amplitude of the external load strongly influences on the values and the behaviour of the electric gradient.

Figure 4 shows the left and right interface debond lengths in the overlap zone as a function of the external load amplitude. The presence of the temperature and the moisture, as well as the interfacial gap along the interface, changes the behaviour (length) of the interface debonding. 

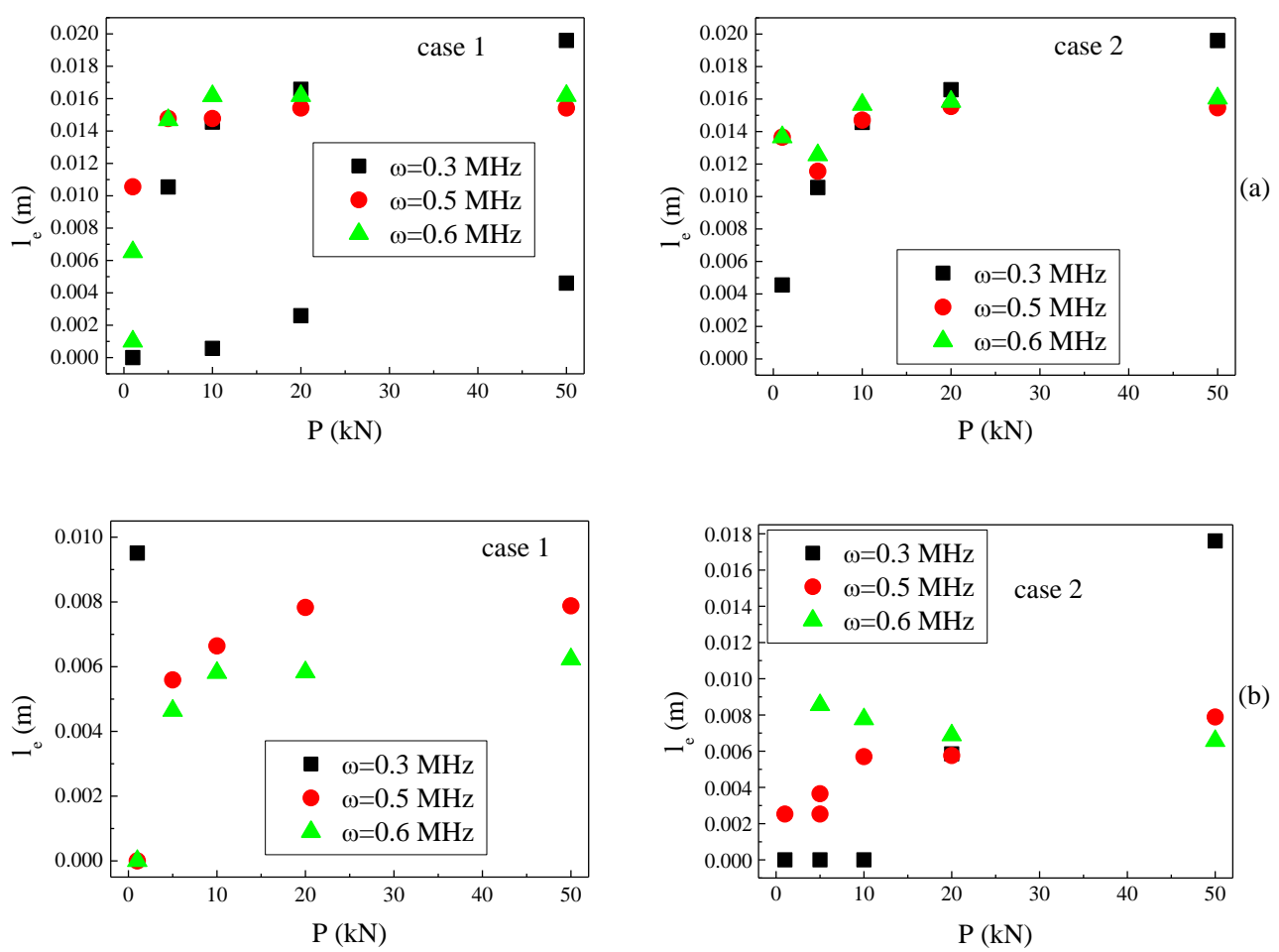

Fig. 4. The debond length vs. mechanical load: (a) for a continuous joint; (b) for a joint with a gap.

\section{CONCLUSIONS}

Two cases of a smart single lap joint (continues and with interfacial gap) are considered, using shear lag analysis. The following conclusions could be derived:

- The presence of the temperature and the moisture, as well as the interfacial gap along the interface, leads to smaller values of the shear stress arises at overlap region;

- The joint with interface gap has a different behaviour, depending on the position of the gap;

- The length of the interface debonding of a joint with gap is smaller compare with continuous joint;

- The temperature and the moisture more strongly influence on the shear stress and electrical gradient. 


\section{ACKNOWLEDGMENTS}

The study has been supported by the National Science Fund, Project DFNI E02/10 121214 .

\section{REFERENCES}

[1] Goland, M., E. Reissner. The Stress in Cemented Joints. J. Appl. Mech., (1944), A17-A26.

[2] Pahoja, H. M. Stress Analysis of an Adhesive Lap Joint Subjected to Tension, Shear Force and Bending Moments, University of Illinois of Urbana-Champaign, T. \& A. M-Report 36 1, August, 1972.

[3] Vinson, J. R. Adhesive Bonding of Polymer Composites. Polym. Eng. Sci., 29 (1989), 1325-1331.

[4] AdAms, R. D. The Mechanics of Bonded Joints, Structural Adhesives in Engineering, ImechE Conference Publications, Suffolk, 1986, 17-24.

[5] AdAms, R. D. Strength Predictions for Lap Joints Especially with Composite Adherents: A Review. J. Adhesion, 30 (1989), 219-242.

[6] Barthelemy, B. M., M. P. Karnat, H. F. Brinson. Finite Element Analysis of Bonded Joints, M. Thesis, Virginia Polytechnic Institute and State University, Virginia, Blacksburg, May, 1984.

[7] Harris, J. A., R. D. Adams. Strength Prediction for Bonded Single Lap Joints by Non-linear Finite Element Methods. Int. J. Adhes. Adhes., 4 (1984), No. 2, 65-78.

[8] Adams, R. D., R. W. AtKins, J. A. Harris. Stress Analysis and Failure Properties of Carbon-Fibre-Reinforced-Plastic Steel Double Lap Joints. J. Adhesion, 20 (1986), 29-53.

[9] KaIrouz, K. C., P. CooK. The Influence of Bond Line Thickness and Overlap Length on the Strength of Bonded Double Lap Composite Joints, Proceedings of ECCM-8 European Conference on Composite Materials, Italy, Naples, June 3-6 1998, 1 (1998), 31-38.

[10] Tsai, M. Y., I. Morton. Experimental and Numerical Studies of a Laminated Composite Single-lap Adhesive Joint. J. Compos. Mater. 29 (1995), No. 9, 527-538.

[11] Bogy, D. B. Edge-bonded Dissimilar Orthogonal Elastic Wedges under Nod and Sheer Loading. J. Appl. Mech., 35 (1968), 460-466.

[12] Dunders, J. Discussion of Edge-bonded Dissimilar Orthogonal Elastic Wedges under Normal and Sheer Loading. J. Appl.Mech., 36 (1969), 650-652.

[13] Bogy, D. B. Two Edge-bonded Elastic Wedges of Different Materials and Wedge Angles under Surface Tractions. J. Appl. Mech., 38 (1972), 377-386.

[14] Erdogan, F. Stress Singularities in a Two-material Wedge. Int. J. Fracture Mech., 7 (1971), 317-330.

[15] Theocan's, P. S., The Order of Singularity at a Multi-Wedge Corner of Composite Plate. Int. J. Eng. Sci., 12 (1974), 107-120. 
[16] Vaidya, U. K., A. R. S. Gautam, M. Hosur, P. Dutta. Experimental-Numerical Studies of Transverse Impact Response of Adhesively Bonded Lap Joints in Composite Structures. Int. J. of Adhesion and Adhesives, 26 (2006), 184-198.

[17] Li, G., P. Lee-Sullivan. Finite Element and Experimental Studies on Single-lap Balanced Joints in Tension. Int. J. of Adhesion and Adhesives, 21 (2001), 211-220.

[18] Olia, M., J. N. Rossetos. Analysis of Adhesively Bonded Joints with Gaps Subjected to Bending. Int. J. Solids Struct., 33 (1996), 2681-2693.

[19] Chadegani, A., R. C. BAtra. Analysis of Adhesive-bonded Single-lap Joint with an Interfacial Crack and a Void. Int. J. of Adhesion and Adhesives, 31 (2011), 455-465.

[20] Becker, W., V. Valeva, T. Petrova, J. Ivanova. Technical Damage in Wind Rotor Blade under Static Load at Environment Conditions. Chemical Engineering Transaction (CET), 42 (2014), 91-96.

\section{APPENDIX}

Using some notations, the expressions for constants in the solutions are given bellow:

$$
\begin{aligned}
F & =\frac{1}{p c}\left[b \frac{\left(D_{A}+\alpha_{A} T\right)}{E_{A}}-k\left(\frac{P}{w E_{B} h_{B}}+\frac{\left(\alpha_{B} T+\beta_{B} H\right)}{E_{B}}\right)\right] \\
Q & =\frac{1}{q c}\left[k\left(\frac{P}{w E_{B} h_{B}}+\frac{\left(\alpha_{B} T+\beta_{B} H\right)}{E_{B}}\right)-a \frac{\left(D_{A}+\alpha_{A} T\right)}{E_{A}}\right] \\
R & =\frac{1}{p c}\left[b\left(\frac{P}{w E_{A} h_{A}}+\frac{\left(D_{A}+\alpha_{A} T\right)}{E_{A}}\right)-k \frac{\left(\alpha_{B} T+\beta_{B} H\right)}{E_{B}}\right] \\
S & =\frac{1}{q c}\left[k \frac{\left(\alpha_{B} T+\beta_{B} H\right)}{E_{B}}-a\left(\frac{P}{w E_{A} h_{A}}+\frac{\left(D_{A}+\alpha_{A} T\right)}{E_{A}}\right)\right],
\end{aligned}
$$

the obtained expressions for constants in the solution, are as follows:

$$
A_{1}^{(2)}=\frac{K_{1} N_{2}-K_{2} M_{2}}{M_{1} N_{2}-M_{2} N_{1}} ; \quad A_{2}^{(2)}=\frac{K_{2} M_{1}-K_{1} N_{1}}{M_{1} N_{2}-M_{2} N_{1}} .
$$

SOLUTION 1: $a=\left(k-\xi-p^{2}\right), b=\left(k-\xi-q^{2}\right), c=\left(p^{2}-q^{2}\right)$.

$$
\begin{array}{ll}
D_{1}=\frac{r\left(\xi+q^{2}\right)}{p c} \frac{\cosh \left(p l_{3}\right)}{\sinh \left(p l_{3}\right)}, & D_{2}=\frac{r\left(\xi+q^{2}\right)}{p c} \frac{\cosh \left(p l_{1}\right)}{\sinh \left(p l_{1}\right)} \\
D_{3}=\frac{r\left(\xi+p^{2}\right)}{q c} \frac{\cosh \left(q l_{3}\right)}{\sinh \left(q l_{3}\right)}, & D_{4}=\frac{r\left(\xi+p^{2}\right)}{q c} \frac{\cosh \left(q l_{1}\right)}{\sinh \left(q l_{1}\right)}
\end{array}
$$




$$
\begin{aligned}
& M_{1}=-p^{2}\left[D_{1} \sin \left(r l_{2}\right)+D_{2} \sin \left(r l_{1}\right)\right]+q^{2}\left[D_{3} \sin \left(r l_{2}\right)+D_{4} \sin \left(r l_{1}\right)\right] \\
& +\xi\left[\cos \left(r l_{2}\right)-\cos \left(r l_{1}\right)\right] \text {, } \\
& M_{2}=p^{2}\left[D_{1} \cos \left(r l_{2}\right)+D_{2} \cos \left(r l_{1}\right)\right]-q^{2}\left[D_{3} \cos \left(r l_{2}\right)+D_{4} \cos \left(r l_{1}\right)\right] \\
& +\xi\left[\sin \left(r l_{2}\right)-\sin \left(r l_{1}\right)\right] \text {, } \\
& K_{1}=-p^{2}\left(\frac{F}{\sinh \left(p l_{1}\right)}+\frac{R}{\sinh \left(p l_{3}\right)}\right)-q^{2}\left(\frac{Q}{\sinh \left(q l_{1}\right)}+\frac{S}{\sinh \left(q l_{3}\right)}\right) \\
& -(\kappa-\xi) l_{g} \frac{\left(D_{A}+\alpha_{A} T\right)}{E_{A}}+k l_{g} \frac{\left(\alpha_{B} T+\beta_{B} H\right)}{E_{B}}, \\
& N_{1}=-a p^{2}\left[D_{1} \sin \left(r l_{2}\right)+D_{2} \sin \left(r l_{1}\right)\right]+b q^{2}\left[D_{3} \sin \left(r l_{2}\right)+D_{4} \sin \left(r l_{1}\right)\right] \\
& +\kappa \eta\left[\cos \left(r l_{2}\right)-\cos \left(r l_{1}\right)\right], \\
& N_{2}=a p^{2}\left[D_{1} \cos \left(r l_{2}\right)+D_{2} \cos \left(r l_{1}\right)\right]-b q^{2}\left[D_{3} \cos \left(r l_{2}\right)+D_{4} \cos \left(r l_{1}\right)\right] \\
& +\kappa \eta\left[\sin \left(r l_{2}\right)-\sin \left(r l_{1}\right)\right] \\
& K_{2}=-a p^{2}\left(\frac{F}{\sinh \left(p l_{1}\right)}+\frac{R}{\sinh \left(p l_{3}\right)}\right)-b q^{2}\left(\frac{Q}{\sinh \left(q l_{1}\right)}+\frac{S}{\sinh \left(q l_{3}\right)}\right) \\
& +\kappa l_{g} \lambda \frac{\left(D_{A}+\alpha_{A} T\right)}{E_{A}}-\kappa l_{g}(\lambda-\eta) \frac{\left(\alpha_{B} T+\beta_{B} H\right)}{E_{B}}, \\
& A_{1}=\frac{1}{\sinh (p l)}[R-F \cosh (p l)], \quad A_{2}=F, \\
& A_{3}=\frac{1}{\sinh (q l)}[S-Q \cosh (q l)], \quad A_{4}=Q, \\
& A_{1}^{(1)}=-\frac{r\left(\xi+q^{2}\right)}{p c} \frac{1}{\sinh \left(p l_{1}\right)}\left[-A_{1}^{(2)} \sin \left(r l_{1}\right)+A_{2}^{(2)} \cos \left(r l_{1}\right)\right]-F \frac{\cosh \left(p l_{1}\right)}{\sinh \left(p l_{1}\right)}, \\
& A_{3}^{(1)}=\frac{r\left(\xi+p^{2}\right)}{q c} \frac{1}{\sinh \left(q l_{1}\right)}\left[-A_{1}^{(2)} \sin \left(r l_{1}\right)+A_{2}^{(2)} \cos \left(r l_{1}\right)\right]-Q \frac{\cosh \left(q l_{1}\right)}{\operatorname{sh}\left(q l_{1}\right)}, \\
& A_{2}^{(1)}=F, \quad A_{4}^{(1)}=Q, \\
& A_{1}^{(3)}=\frac{r\left(\xi+q^{2}\right)}{p c} \frac{\cosh (p l)}{\sinh \left(p l_{3}\right)}\left[-A_{1}^{(2)} \sin \left(r l_{2}\right)+A_{2}^{(2)} \cos \left(r l_{2}\right)\right]+R \frac{\cosh \left(p l_{2}\right)}{\sinh \left(p l_{3}\right)}, \\
& A_{2}^{(3)}=-\frac{r\left(\xi+q^{2}\right)}{p c} \frac{\sinh (p l)}{\sinh \left(p l_{3}\right)}\left[-A_{1}^{(2)} \sin \left(r l_{2}\right)+A_{2}^{(2)} \cos \left(r l_{2}\right)\right]-R \frac{\sinh \left(p l_{2}\right)}{\sinh \left(p l_{3}\right)}, \\
& A_{3}^{(3)}=-\frac{r\left(\xi+p^{2}\right)}{q c} \frac{\cosh (q l)}{\sinh \left(q l_{3}\right)}\left[-A_{1}^{(2)} \sin \left(r l_{2}\right)+A_{2}^{(2)} \cos \left(r l_{2}\right)\right]+S \frac{\cosh \left(q l_{2}\right)}{\sinh \left(q l_{3}\right)}, \\
& A_{4}^{(3)}=\frac{r\left(\xi+p^{2}\right)}{q c} \frac{\sinh (q l)}{\sinh \left(q l_{3}\right)}\left[-A_{1}^{(2)} \sin \left(r l_{2}\right)+A_{2}^{(2)} \cos \left(r l_{2}\right)\right]+S \frac{\sinh \left(q l_{2}\right)}{\sinh \left(q l_{3}\right)} .
\end{aligned}
$$


SOLUTION 2: $a=\left(k-\xi-p^{2}\right), b=\left(k-\xi+q^{2}\right), c=\left(p^{2}+q^{2}\right)$

$$
\begin{array}{ll}
D_{1}=\frac{r\left(\xi-q^{2}\right)}{p c} \frac{\cosh \left(p l_{3}\right)}{\sinh \left(p l_{3}\right)}, & D_{2}=\frac{r\left(\xi-q^{2}\right)}{p c} \frac{\cosh \left(p l_{1}\right)}{\sinh \left(p l_{1}\right)} \\
D_{3}=\frac{r\left(\xi+p^{2}\right)}{q c} \frac{\cos \left(q l_{3}\right)}{\sin \left(q l_{3}\right)}, & D_{4}=\frac{r\left(\xi+p^{2}\right)}{q c} \frac{\cos \left(q l_{1}\right)}{\sin \left(q l_{1}\right)}
\end{array}
$$

$$
\begin{aligned}
M_{1}= & -p^{2}\left[D_{1} \sin \left(r l_{2}\right)+D_{2} \sin \left(r l_{1}\right)\right]+q^{2}\left[D_{3} \sin \left(r l_{2}\right)+D_{4} \sin \left(r l_{1}\right)\right] \\
& +\xi\left[\cos \left(r l_{2}\right)-\cos \left(r l_{1}\right)\right], \\
M_{2}= & p^{2}\left[D_{1} \cos \left(r l_{2}\right)+D_{2} \cos \left(r l_{1}\right)\right]-q^{2}\left[D_{3} \cos \left(r l_{2}\right)+D_{4} \cos \left(r l_{1}\right)\right] \\
& +\xi\left[\sin \left(r l_{2}\right)-\sin \left(r l_{1}\right)\right], \\
K_{1}= & -p^{2}\left(\frac{F}{\sinh \left(p l_{1}\right)}+\frac{R}{\sinh \left(p l_{3}\right)}\right)-q^{2}\left(\frac{Q}{\sin \left(q l_{1}\right)}+\frac{S}{\sin \left(q l_{3}\right)}\right) \\
& -(\kappa-\xi) l_{g} \frac{\left(D_{A}+\alpha_{A} T\right)}{E_{A}}+k l_{g} \frac{\left(\alpha_{B} T+\beta_{B} H\right)}{E_{B}}, \\
N_{1}= & -a p^{2}\left[D_{1} \sin \left(r l_{2}\right)+D_{2} \sin \left(r l_{1}\right)\right]+b q^{2}\left[D_{3} \sin \left(r l_{2}\right)+D_{4} \sin \left(r l_{1}\right)\right] \\
& +\kappa \eta\left[\cos \left(r l_{2}\right)-\cos \left(r l_{1}\right)\right], \\
N_{2}= & a p^{2}\left[D_{1} \cos \left(r l_{2}\right)+D_{2} \cos \left(r l_{1}\right)\right]-b q^{2}\left[D_{3} \cos \left(r l_{2}\right)+D_{4} \cos \left(r l_{1}\right)\right] \\
& +\kappa \eta\left[\sin \left(r l_{2}\right)-\sin \left(r l_{1}\right)\right], \\
K_{2}= & -a p^{2}\left(\frac{F}{\sinh \left(p l_{1}\right)}+\frac{R}{\sinh \left(p l_{3}\right)}\right)-b q^{2}\left(\frac{Q}{\sin \left(q l_{1}\right)}+\frac{S}{\sin \left(q l_{3}\right)}\right) \\
& +\kappa l_{g} \lambda \frac{\left(D_{A}+\alpha_{A} T\right)}{E_{A}}-\kappa l_{g}(\lambda-\eta) \frac{\left(\alpha_{B} T+\beta_{B} H\right)}{E_{B}},
\end{aligned}
$$

$A_{1}=\frac{1}{\sinh (p l)}[R-F \cosh (p l)], \quad A_{2}=F$,

$A_{3}=\frac{1}{\sin (q l)}[Q \cos (q l)-S], \quad A_{4}=Q$

$A_{1}^{(1)}=-\frac{r\left(\xi-q^{2}\right)}{p c} \frac{1}{\sinh \left(p l_{1}\right)}\left[-A_{1}^{(2)} \sin \left(r l_{1}\right)+A_{2}^{(2)} \cos \left(r l_{1}\right)\right]-F \frac{\cosh \left(p l_{1}\right)}{\sinh \left(p l_{1}\right)}$,

$A_{3}^{(1)}=-\frac{r\left(\xi+p^{2}\right)}{q c} \frac{1}{\sinh \left(q l_{1}\right)}\left[-A_{1}^{(2)} \sin \left(r l_{1}\right)+A_{2}^{(2)} \cos \left(r l_{1}\right)\right]+Q \frac{\cosh \left(q l_{1}\right)}{\sinh \left(q l_{1}\right)}$,

$A_{2}^{(1)}=F, \quad A_{4}^{(1)}=Q$

$A_{1}^{(3)}=\frac{r\left(\xi-q^{2}\right)}{p c} \frac{\cosh (p l)}{\sinh \left(p l_{3}\right)}\left[-A_{1}^{(2)} \sin \left(r l_{2}\right)+A_{2}^{(2)} \cos \left(r l_{2}\right)\right]+R \frac{\cosh \left(p l_{2}\right)}{\sinh \left(p l_{3}\right)}$, 


$$
\begin{aligned}
& A_{2}^{(3)}=-\frac{r\left(\xi-q^{2}\right)}{p c} \frac{\sinh (p l)}{\sinh \left(p l_{3}\right)}\left[-A_{1}^{(2)} \sin \left(r l_{2}\right)+A_{2}^{(2)} \cos \left(r l_{2}\right)\right]-R \frac{\sinh \left(p l_{2}\right)}{\sinh \left(p l_{3}\right)}, \\
& A_{3}^{(3)}=\frac{r\left(\xi+p^{2}\right)}{q c} \frac{\cos (q l)}{\sin \left(q l_{3}\right)}\left[-A_{1}^{(2)} \sin \left(r l_{2}\right)+A_{2}^{(2)} \cos \left(r l_{2}\right)\right]-S \frac{\cos \left(q l_{2}\right)}{\sin \left(q l_{3}\right)}, \\
& A_{4}^{(3)}=\frac{r\left(\xi+p^{2}\right)}{q c} \frac{\sin (q l)}{\sin \left(q l_{3}\right)}\left[-A_{1}^{(2)} \sin \left(r l_{2}\right)+A_{2}^{(2)} \cos \left(r l_{2}\right)\right]-S \frac{\sin \left(q l_{2}\right)}{\sin \left(q l_{3}\right)} .
\end{aligned}
$$

SOLUTION 3: $a=\left(k-\xi+p^{2}\right), b=\left(k-\xi+q^{2}\right), c=\left(p^{2}-q^{2}\right)$

$$
\begin{array}{ll}
D_{1}=\frac{r\left(\xi-q^{2}\right)}{p c} \frac{\cos \left(p l_{3}\right)}{\sin \left(p l_{3}\right)}, & D_{2}=\frac{r\left(\xi-q^{2}\right)}{p c} \frac{\cos \left(p l_{1}\right)}{\sin \left(p l_{1}\right)}, \\
D_{3}=\frac{r\left(\xi-p^{2}\right)}{q c} \frac{\cos \left(q l_{3}\right)}{\sin \left(q l_{3}\right)}, & D_{4}=\frac{r\left(\xi-p^{2}\right)}{q c} \frac{\cos \left(q l_{1}\right)}{\sin \left(q l_{1}\right)},
\end{array}
$$

$$
\begin{aligned}
M_{1}= & -p^{2}\left[D_{1} \sin \left(r l_{2}\right)+D_{2} \sin \left(r l_{1}\right)\right]+q^{2}\left[D_{3} \sin \left(r l_{2}\right)+D_{4} \sin \left(r l_{1}\right)\right] \\
& -\xi\left[\cos \left(r l_{2}\right)-\cos \left(r l_{1}\right)\right] \\
M_{2}= & p^{2}\left[D_{1} \cos \left(r l_{2}\right)+D_{2} \cos \left(r l_{1}\right)\right]-q^{2}\left[D_{3} \cos \left(r l_{2}\right)+D_{4} \cos \left(r l_{1}\right)\right] \\
& -\xi\left[\sin \left(r l_{2}\right)-\sin \left(r l_{1}\right)\right] \\
K_{1}= & p^{2}\left(\frac{F}{\sin \left(p l_{1}\right)}+\frac{R}{\sin \left(p l_{3}\right)}\right)+q^{2}\left(\frac{Q}{\sin \left(q l_{1}\right)}+\frac{S}{\sin \left(q l_{3}\right)}\right) \\
& +(\kappa-\xi) l_{g} \frac{\left(D_{A}+\alpha_{A} T\right)}{E_{A}}-k l_{g} \frac{\left(\alpha_{B} T+\beta_{B} H\right)}{E_{B}}, \\
N_{1}= & -a p^{2}\left[D_{1} \sin \left(r l_{2}\right)+D_{2} \sin \left(r l_{1}\right)\right]+b q^{2}\left[D_{3} \sin \left(r l_{2}\right)+D_{4} \sin \left(r l_{1}\right)\right] \\
& -\kappa \eta\left[\cos \left(r l_{2}\right)-\cos \left(r l_{1}\right)\right], \\
N_{2}= & a p^{2}\left[D_{1} \cos \left(r l_{2}\right)+D_{2} \cos \left(r l_{1}\right)\right]-b q^{2}\left[D_{3} \cos \left(r l_{2}\right)+D_{4} \cos \left(r l_{1}\right)\right] \\
& -\kappa \eta\left[\sin \left(r l_{2}\right)-\sin \left(r l_{1}\right)\right], \\
K_{2}= & a p^{2}\left(\frac{F}{\sin \left(p l_{1}\right)}+\frac{R}{\sin \left(p l_{3}\right)}\right)+b q^{2}\left(\frac{Q}{\sin \left(q l_{1}\right)}+\frac{\left(\alpha_{1}\left(q l_{3}\right)\right.}{\sin }\right) \\
& -\kappa l_{g} \lambda \frac{\left(D_{A}+\alpha_{A} T\right)}{E_{A}}+\kappa l_{g}(\lambda-\eta) \frac{\left(\alpha_{B} T+\beta_{B} H\right)}{E_{B}} \\
A_{1}= & \frac{1}{\sin (p l)}[F \cos (p l)-R], \quad A_{2}=F, \\
A_{3}= & \frac{1}{\sin (q l)}[Q \cos (q l)-S], \quad A_{4}=Q \\
A_{1}^{(1)}= & -\frac{r\left(\xi-q^{2}\right)}{p c} \frac{1}{\sin \left(p l_{1}\right)}\left[-A_{1}^{(2)} \sin \left(r l_{1}\right)+A_{2}^{(2)} \cos \left(r l_{1}\right)\right]+F \frac{\cos \left(p l_{1}\right)}{\sin \left(p l_{1}\right)}
\end{aligned}
$$




$$
\begin{aligned}
& A_{3}^{(1)}=\frac{r\left(\xi-p^{2}\right)}{q c} \frac{1}{\sin \left(q l_{1}\right)}\left[-A_{1}^{(2)} \sin \left(r l_{1}\right)+A_{2}^{(2)} \cos \left(r l_{1}\right)\right]+Q \frac{\cos \left(q l_{1}\right)}{\sin \left(q l_{1}\right)}, \\
& A_{2}^{(1)}=F, \quad A_{4}^{(1)}=Q, \\
& A_{1}^{(3)}=\frac{r\left(\xi-q^{2}\right)}{p c} \frac{\cos (p l)}{\sin \left(p l_{3}\right)}\left[-A_{1}^{(2)} \sin \left(r l_{2}\right)+A_{2}^{(2)} \cos \left(r l_{2}\right)\right]-R \frac{\cos \left(p l_{2}\right)}{\sin \left(p l_{3}\right)}, \\
& A_{2}^{(3)}=\frac{r\left(\xi-q^{2}\right)}{p c} \frac{\sin (p l)}{\sin \left(p l_{3}\right)}\left[-A_{1}^{(2)} \sin \left(r l_{2}\right)+A_{2}^{(2)} \cos \left(r l_{2}\right)\right]-R \frac{\sin \left(p l_{2}\right)}{\sin \left(p l_{3}\right)} \\
& A_{3}^{(3)}=\frac{r\left(\xi-p^{2}\right)}{q c} \frac{\cos (q l)}{\sin \left(q l_{3}\right)}\left[-A_{1}^{(2)} \sin \left(r l_{2}\right)+A_{2}^{(2)} \cos \left(r l_{2}\right)\right]-S \frac{\cos \left(q l_{2}\right)}{\sin \left(q l_{3}\right)} \\
& A_{4}^{(3)}=\frac{r\left(\xi-p^{2}\right)}{q c} \frac{\sin (q l)}{\sin \left(q l_{3}\right)}\left[-A_{1}^{(2)} \sin \left(r l_{2}\right)+A_{2}^{(2)} \cos \left(r l_{2}\right)\right]-S \frac{\sin \left(q l_{2}\right)}{\sin \left(q l_{3}\right)}
\end{aligned}
$$

